\title{
New COMPASS results on the proton spin-dependent structure function $g_{1}^{p}$
}

\author{
Vincent ANDRIEUX* \\ On behalf of the COMPASS Collaboration \\ CEA Saclay, DSM/IRFU/SPhN \\ F91191 Gif-sur-Yvette, France \\ E-mail: vincent.andrieux@cern.ch
}

Recent COMPASS results on the spin asymmetry $A_{1}$ and the spin-dependent structure function of the proton $g_{1}^{p}$ as a function of $x_{B j}$ and $Q^{2}$ are presented. The data have been recorded with a polarised muon beam at $200 \mathrm{GeV}$ scattered off a polarised $\mathrm{NH}_{3}$ target. The high energy of the beam allows for $A_{1}$ measurements down to $x_{B j}=0.0036$ for the first time and extends the $Q^{2}$ range to higher values, which brings new inputs for QCD global fits of world data.

XXI International Workshop on Deep-Inelastic Scattering and Related Subjects 22-26 April, 2013

Marseille, France

${ }^{*}$ Speaker. 


\section{Introduction}

The COMPASS spectrometer [1], located at CERN, is dedicated to the study of spin structure of the nucleon and hadron spectroscopy using the SPS beam. We present here new results on the proton spin-dependent structure function extracted from deep inelastic scattering (DIS). A polarised muon beam with an energy of $200 \mathrm{GeV}$ is scattered off a longitudinally polarised $\mathrm{NH}_{3}$ target. These new data complement the previous COMPASS proton results measured at $160 \mathrm{GeV}$ [2]. The increase of the beam energy allows us to explore a wider kinematic domain. It contributes to a better knowledge of the scaling violation of $g_{1}^{p}$ which constitutes an indirect measurement of $\Delta G$. This new set of data improves the statistics on the spin dependent structure function in the whole $x_{B j}$ range of measurement and especially in the low $x_{B j}$ region. The kinematic domain covered by the data is extended to $100(\mathrm{GeV} / \mathrm{c})^{2}$ in $Q^{2}$ and down to 0.0036 in $x_{B j}$. Figure 1a shows the available kinematic domain and the effect of the rise of the energy.

\section{Measurement \& Event Selection}

The data were recorded in 2011. The muon beam comes mostly from the weak decay of beam pions and is hence naturally polarised. Its polarisation is $80 \%$. The target is embedded in a solenoid producing a $2.5 \mathrm{~T}$ field. The ammonia which composes the target material is then polarised by dynamic nuclear polarisation. The target structure has been optimised to record simultaneously data with the two spin states, therefore it has been divided into three cells with the central one polarised oppositely to its two nearby cells. The track of the incoming muon is required to cross all the cells to have the same flux for each cell. Moreover the central cell is twice as long as the upstream and downstream cells to keep the same acceptance for the two spin state on average. The $\mathrm{NH}_{3}$ polarisation is about $85 \%$ with a fraction of polarisable nucleons of $16 \%$. The DIS regime is assured by a cut on $Q^{2}>1 \mathrm{GeV} / \mathrm{c}^{2}$. Only events with a photon fraction of the energy, y, within the range [0.1,0.9] are kept. This selection removes events which are hardly reconstructible $(y<0.1)$ and the region the most affected by the radiative corrections $(y>0.9)$.

\section{Spin asymmetry, $A_{1}^{p}$, and spin structure function, $g_{1}^{p}$, extraction}

The asymmetry of longitudinally polarised cross-section $A_{\|}^{p}=\left(\sigma^{\uparrow \downarrow}-\sigma^{\uparrow \uparrow}\right) /\left(\sigma^{\uparrow \downarrow}+\sigma^{\uparrow \uparrow}\right)$ is related to a combination of the virtual photon-nucleon asymmetries $A_{1}$ and $A_{2}$ weighted by some kinematic factor: $A_{\|}^{p}=D\left(A_{1}+\eta A_{2}\right)$. The $\eta$ factor is always small within the COMPASS kinematic, moreover $A_{2}$ has been found to be small [3], thus the $\eta A_{2}$ term is neglected. Hence $A_{1}^{p}$ and $g_{1}^{p}$ are expressed as :

$$
A_{\|} \simeq D A_{1} \quad \text { (3.1) } \quad g_{1}^{p}=\frac{F_{2}^{p}}{2 x_{B j}(1+R)} A_{1}^{p}
$$

where $\mathrm{D}$ is the depolarisation factor. $F_{2}^{p}$ and $\mathrm{R}$ are spin-independent structure functions taken from [4] and [5] respectively. The data have been corrected for radiative effects [6], [7]. A correction for ${ }^{14} \mathrm{~N}$ contribution in the target material has also been performed according to a model described in [8]. 


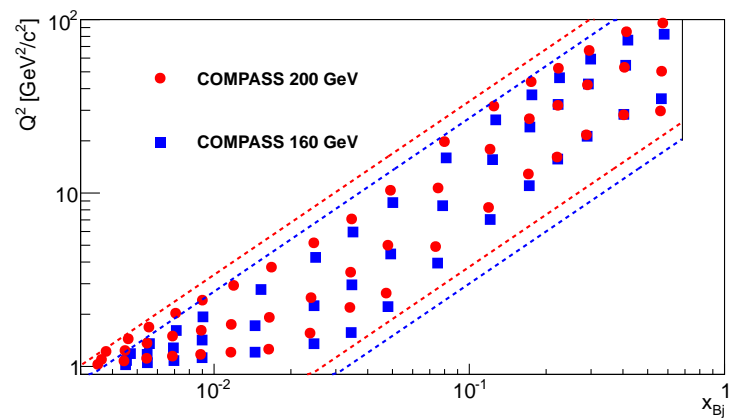

(a)

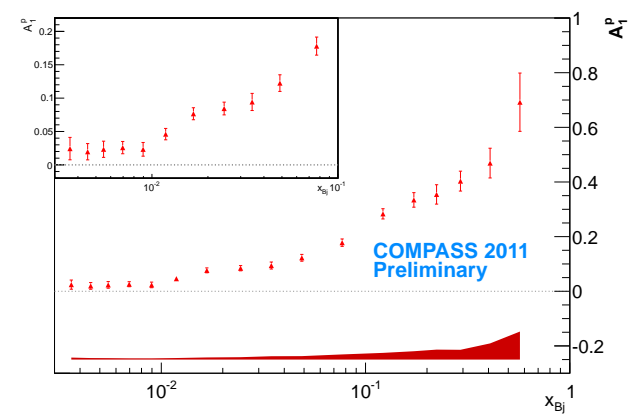

(b)

Figure 1: (a): The mean values of $Q^{2}$ versus the mean values of $x_{B j}$ for the new COMPASS at 200 $\mathrm{GeV}$ and compared to previous COMPASS data at $160 \mathrm{GeV}$. (b): $A_{1}^{p}$ as a function of $x_{B j}$ for the COMPASS. The bottom band shows the systematic errors.

\section{Results}

The proton spin asymmetry $A_{1}^{p}$ measured by COMPASS at $200 \mathrm{GeV}$ is shown as function of $x_{B j}$ in Fig. 1b. The corresponding proton spin structure function $g_{1}^{p}$ for COMPASS $200 \mathrm{GeV}$ is shown versus $x_{B j}$ and integrated over $Q^{2}$ in Fig. 2. The results from the previous COMPASS and SMC proton data are also shown.

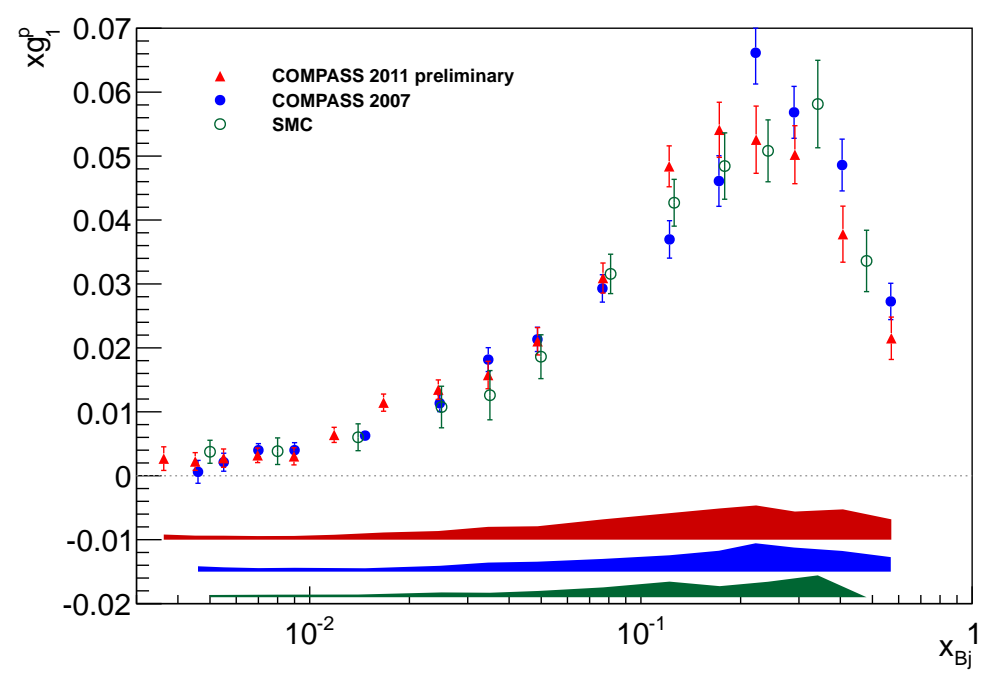

Figure 2: The proton structure function $x g_{1}^{p}$ as measured at COMPASS at $200 \mathrm{GeV}$ is plotted as a function of $x_{B j}$. The previous results from COMPASS $160 \mathrm{GeV}$ and SMC are also shown for comparison. COMPASS $200 \mathrm{GeV}$ systematics are the top band, followed by COMPASS $160 \mathrm{GeV}$ and SMC $190 \mathrm{GeV}$. 
For the new $200 \mathrm{GeV}$ data, the main components of the preliminary systematic errors come from the beam polarisation, the target polarisation and the limit to a possible false asymmetry. The latter is estimated by a statistical test performed on the distribution of asymmetries extracted from 78 subsamples. For each $x_{B j}$ value the asymmetry distribution is fitted to a Gaussian distribution and the width parameter with its error are used to set a limit to the false asymmetry. All the results are consistent with each other and the COMPASS data improve the statistical accuracy by a factor two to three in comparison with the SMC data. Furthermore, an additional point at very low $x_{B j}$ has been measured and brings more constraints in the fit and the determination of the integral of $g_{1}$ which the low $x_{B j}$ region is the greatest source of uncertainties.

To better make use of the wider kinematic domain, each x-bin of $A_{1}^{p}$ has been split into three bins of $Q^{2}$ and shown in Fig. 3.

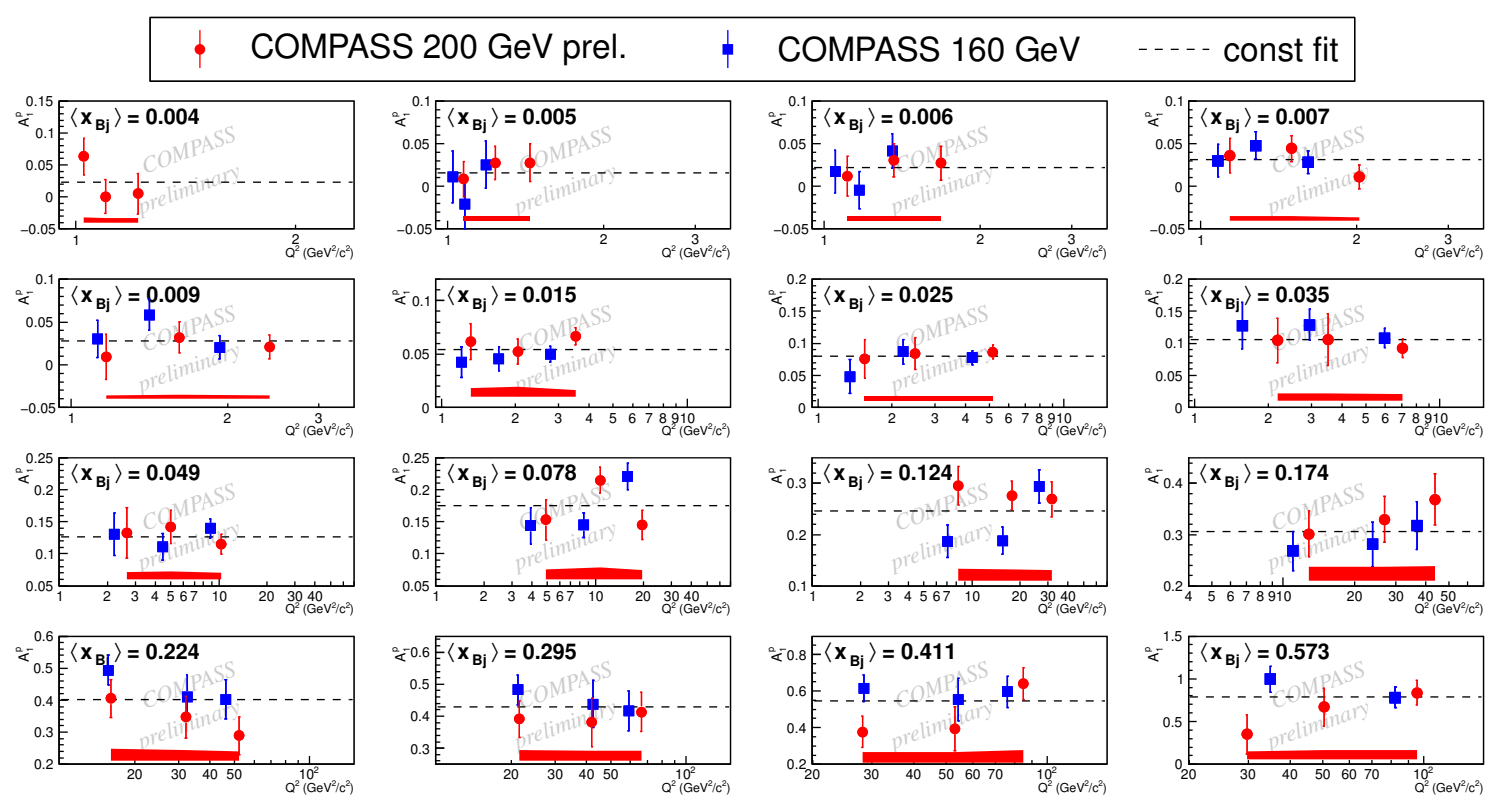

Figure 3: COMPASS $A_{1}^{P}$ results versus $Q^{2}$ for the different $x_{B j}$-bin. Only the systematics for 200 $\mathrm{GeV}$ data are shown by the band at the bottom of each pad. The upper left pad shows only 200 $\mathrm{GeV}$ data since its value of $x_{B j}$ has been accessed for the first time. The dashed lines show a fit of the data points to a constant.

The results of the world measurement of the proton spin structure function as a function of $Q^{2}$ for different values of $x_{B j}$ are shown in the Fig. 4. The data points for the different experiments are slightly readjusted to the same value of $x_{B j}$ according to formula 4.1, based on the LSS parametrisation of world data [9].

$$
g_{1}^{p}\left(x_{B j}, Q_{\text {meas }}^{2}\right)=g_{1(\text { data })}^{p}+g_{1(\text { LSS })}^{p}\left(x_{B j}, Q_{\text {meas }}^{2}\right)-g_{1(\text { LSS })}^{p}\left(x_{B j}^{\text {meas }}, Q_{\text {meas }}^{2}\right)
$$




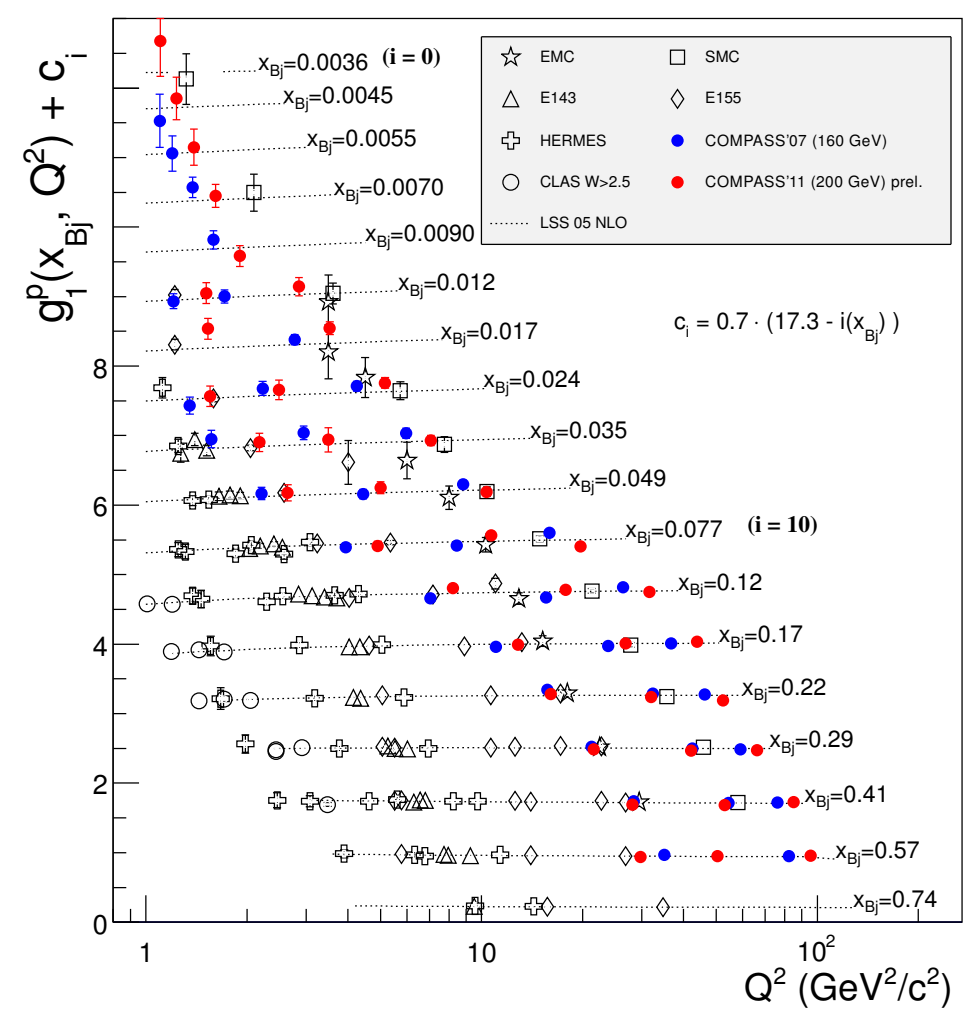

Figure 4: World data measurements of the proton spin structure function versus $Q^{2}$ in bins of $x_{B j}$. The dashed line shows the LSS05 fit at NLO.

\section{Summary}

New results of the proton spin structure function $g_{1}^{p}$ measured at COMPASS at $200 \mathrm{GeV}$ have been presented. They complete the previously existing data at $160 \mathrm{GeV}$, enlarging the $x_{B j}$ and $Q^{2}$ coverage. In most $x_{B j}$, they provide a result at the highest $Q^{2}$ value. They constitute an important input for global QCD fits at NLO.

\section{References}

[1] COMPASS, P. Abbon et al., Nucl. Instrum. Methods. A 577 (2007) 455.

[2] COMPASS, M.G. Alekseev et al., Phys. Lett. B 690 (2010) 466.

[3] P.L. Anthony et al., Phys. Lett. B 553 (2003) 18.

[4] SMC, B. Adeva et al., Phys. Rev. D 58 (1998) 112001.

[5] COMPASS, V.Yu. Alexakhin et al., Phys. Lett. B 647 (2007) 330.

[6] A. A. Akhundov et al., Fortsch. Phys. 44 (1996) 373.

[7] I. Akushevich et al., Computer physics communications 104 (1997) 201.

[8] O. A. Rondon, Phys. Rev. C 60 (1999) 035201.

[9] E. Leader et al., Phys. Rev. D 73 (2006) 034023 